\title{
Study of Photoemission from BAs by Using Muffin-Tin
}

\section{Potential}

\author{
Rebecca Lalngaihawmi ${ }^{1}$, Benjamin Vanlalruata ${ }^{1}$, M. P. Ghimire ${ }^{2,3}$, R. K. Thapa ${ }^{1,2^{*}}$ and A. H. Reshak ${ }^{4,5}$ \\ 1. Condensed Matter Theory Research Group, Department of Physics, Mizoram University, 796004, India \\ 2. Condensed Matter Physics Research Center, Butwal, Rupendehi, Nepal \\ 3. IFW Dresden, Helmholtz Straße 20, 01069 Dresden, Germany \\ 4. New Technologies - Research Centre, University of West Bohemia, Univerzitni 8, 30614 Pilsen, Czech Republic \\ 5. Center of Excellence Geopolymer and Green Technology, School of Material Engineering, University Malaysia \\ Perlis, 01007 Kangar, Perlis, Malaysia
}

\begin{abstract}
We here present the results of photocurrent from BAs. The photocurrent in this system had been calculated by using the free-electron and muffin-tin potentials. Schrodingers' equation has been solved by using these potentials to obtain the necessary initial and final state wave-functions for the electrons to evaluate the matrix element for transitions. From the calculations it is observed that variation of photocurrent showed trends obtained as in the case of metals like tungsten, aluminium, molybdenum, copper etc.
\end{abstract}

Key words: Wien2k, DFT (Density functional theory), muffin-tin, FP-LAPW.

\section{Introduction}

Boron Arsenide is a wide band gap semiconductor compound in the III-V family and occurs as cubic sphalerite. This compound crystallizes in zinc-blende structure [1]. It has a number of technological applications such as solar cells, space electronics and devices that produce electrical energy by coupling a radioactive beta emitter to a semiconductor junction. In this paper here, we present study of photoemission from BAs by using the muffin-tin potential model. In photoemission, the incident radiation excites the electrons lying at the Fermi level or below it. The photo-excited electrons gainsnow sufficient energy to overcome the work function of the metal and hence comes out into the vacuum to contribute to photocurrent. For evaluation of the vector potential involved in the matrix element for transition from initial to final state, we require real and imaginary

\footnotetext{
${ }^{*}$ Corresponding author: R. K. Thapa, prof. Dr., research
} field: condensed matter physics. dielectric constants. These had been calculated by using the density functional theory (DFT) based on full-potential approximation which is implemented in wien $2 \mathrm{k}$ code [2].

\section{Methodology}

In our study of photoemission from BAs, the current density for photoemission is calculated by using the golden-rule approximations [3] as:

$$
\begin{aligned}
& \frac{d j(E)}{d \Omega}=\frac{2 \pi}{\hbar c} \sum_{f, i}\left|\left\langle\Psi_{f}\left|H^{\prime}\right| \Psi_{i}\right\rangle\right|^{2} \delta\left(E-E_{F}\right) \delta\left(E_{f}-\right. \\
& \left.E_{i}-\hbar \omega\right) f_{0}(E-\hbar \omega)\left[1-f_{0}(E)\right]
\end{aligned}
$$

where, $\Psi_{i}$ and $\Psi_{f}$ are the initial and final states wave-function and $H^{\prime}=(e / 2 m c)(A \cdot p+p \cdot A), A$ being the vector potential and $p$ the one-electron momentum operator. In order to evaluate the matrix element, we need to construct $\Psi_{i}$ and $\Psi_{f}$ and determine $A$. Although the one-electron states are treated quite accurately in many photoemission calculations [4], the variation of the photon field in the surface region is usually neglected and hence we have 
used a simple model for field calculations as done by Bagchi and Kar [5].

We have used the muffin-tin potential to derive the initial state wave function $\Psi_{i}$ [6] used in Eq. (1), which is given by

$$
\begin{aligned}
& \Psi_{i}(\vec{r}) \\
& =\left\{\begin{array}{c}
\sum_{L} A_{1 L} f_{1 l}(R) Y_{L}(\theta, \phi), \text { spherical region } \\
\sum_{\vec{g}}\left[u_{\vec{g}} e^{i K_{\vec{g}}^{+} \cdot \vec{R}}+V_{\vec{g}} e^{i \vec{K}_{\vec{g}} \cdot \vec{R}}\right], \text { interstitial region }
\end{array}\right.
\end{aligned}
$$

where, $\quad K_{\vec{g}}^{+}=\left[(\vec{k}+\vec{g})_{\|} \pm \sqrt{2\left(E_{i}-V_{0}\right)-|\vec{k}+\vec{g}|_{\|}^{2}}\right]$ and $\vec{R}=\vec{r}-\vec{C}_{j}$.

Similarly, the final state wavefunction [6] can be written as

$$
\Psi_{f}(\vec{r})= \begin{cases}{\left[e^{i g z}+\frac{g-k_{f}}{g+k_{f}} e^{-i g z} e^{i \vec{k}_{1} \vec{r}_{1}}\right] e^{i \vec{k}_{\|} \vec{r}_{\|}}, \quad z>0} \\ \frac{2 g}{g+k_{f}} e^{i k_{f} z} e^{i \vec{k}_{\|} \vec{r}_{\|,}} & z<0\end{cases}
$$

where, $\quad \vec{k}_{f}^{2}=\frac{2 m E_{F}}{\hbar^{2}}-\vec{k}_{\|}^{2}, \quad \vec{g}^{2}=\frac{2 m}{\hbar^{2}}\left(E_{F}-V_{0}\right)-\vec{k}_{\|}^{2}$, $E_{f}=E_{i}+\hbar \omega$.

The initial state wavefunctionused for the free-electron model [7] is given by:

$$
\Psi_{i}(\vec{r})= \begin{cases}{\left[e^{i k_{i} z}+\frac{i k_{i} z+\chi}{i k_{i} z-\chi} e^{-i k_{i} z}\right] e^{-\vec{k}_{\|} \vec{r}_{\|},},} & z<0 \\ \frac{2 i k_{i}}{i k_{i}-\chi} e^{-\chi z} e^{\vec{k}_{\|}} \vec{r}_{\|,}, & z>0\end{cases}
$$

where, $\vec{k}_{||}$and $\vec{r}_{||}$are the components of $\vec{k}$ and $\vec{r}$ in the X-Y plane i.e. the plane parallel to the surface. The final state wavefunction is as given in Eq. 3 .

\section{Results and Discussion}

We have computed our results for normal photoemission from the surface and bulk states of BAs as a function of photon energy. Hence the initial state energy $E_{i}=6.098 \mathrm{eV}, E_{F}=7.319 \mathrm{eV}$, potential energy $V_{0}=15.95 \mathrm{eV}$, work function $\phi=$ $8.631 \mathrm{eV}$, surface width $\mathrm{a}=5.298 \AA$, angle of incident radiation $\theta_{i}=45^{\circ}$ and scattering factor $\alpha=0.35$. For calculations of the electromagnetic fields which is related to vector potential, we have used the real and dielectric constants calculated by using the full-potential linearized augmented plane wave method implemented in Wien2k code [2] which is based on the density functional theory [8].

In Fig. 1, we show the plot of photocurrent as a function of photon energy of the incident radiation from the first and second surface layers of the medium. In this calculation, dielectric constants $\varepsilon_{1}$ and $\varepsilon_{2}$ were obtained by using the Wien2k code with GGA approximation [8]. For the case of photoemission from the first surface layer, we find that photocurrent increases as photon energy increases and this becomes maximum at photon energy $10 \mathrm{eV}$. With the increase in photon energy, we find that photocurrent decreases and showed small humps at photon energies $13 \mathrm{eV}$ and $17 \mathrm{eV}$ photon $\mathrm{eV}$. Photocurrent was minimum at $22 \mathrm{eV}$ incident photon radiation energy. Again, beyond $22 \mathrm{eV}$ energy, the photocurrent showed rise in photocurrent with the increase of incident radiation. The second maxima in photocurrent occurred at $28 \mathrm{eV}$ photon energy after which it decreases gradually.

In Fig. 1, we have plotted photocurrent as a function of photon energy for BAs from the second layer of the system. The trend in photocurrent is different than in the case of photoemission from the

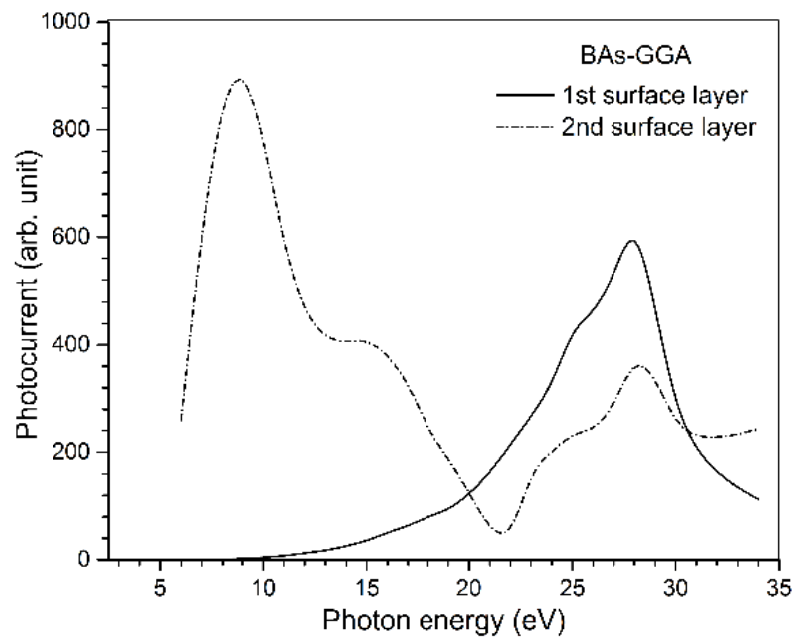

Fig. 1 Plot of photocurrent in BAs as a function of photon energy from the fist two layers. 


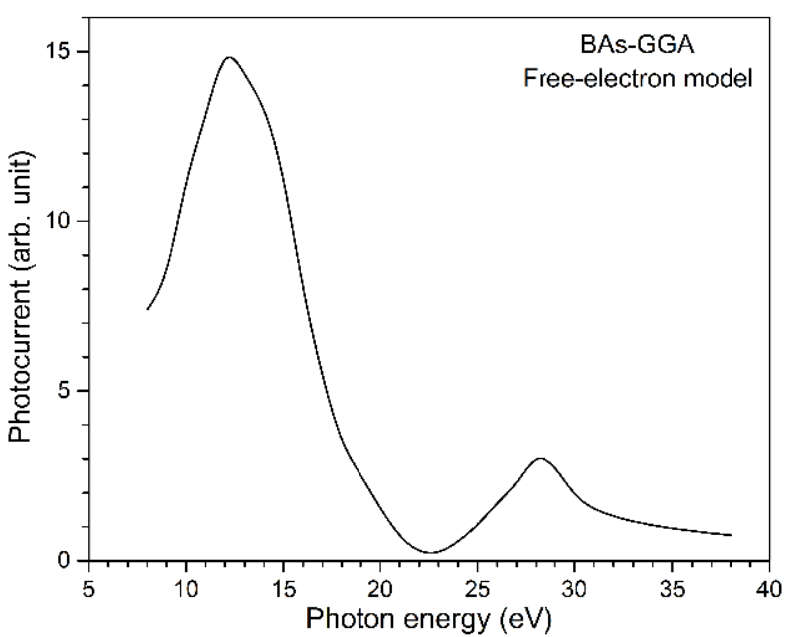

Fig. 2 Plot of photocurrent in BAs as function of photon energy using free-electron-model.

first surface layer. Photocurrent simply rises with an increase of photon energy and at $26 \mathrm{eV}$ photon energy, there is occurrence of peak in photocurrent. Photocurrent decreases with the further increase in photon energy after $26 \mathrm{eV}$ and becomes minimum. Contribution of photocurrent from second layer showed similar trends as from the first layer, but occurrence of peaks are at different photon energies.

In Fig. 2, we show the plot of photocurrent as a function of incident photon energy for the values of same parameters as used above in the case of BAs but by using the free electron potential [9]. The same values ofdielectric constants as used in the case of surface layer calculations above was used. The results of photocurrent here showed features similar to the case of metals like beryllium and aluminium as was obtained by Thapa et al earlier [7, 9, 10]. For example, there is maxima in photocurrent at $12 \mathrm{eV}$ photon energy and photocurrent decreased with the increase of photon energy. At photon energy $22.5 \mathrm{eV}$, there was occurrence of minima in photocurrent. Beyond the increase in photocurrent from $22.5 \mathrm{eV}$, we find there is also an occurrence of second maxima in photocurrent but of very small height comparable to the one at $12 \mathrm{eV}$. After this the photocurrent decreases gradually with the increase in photon energy the features similarly observed in the case beryllium, aluminium etc. [11]
We may conclude that the surface variation of photon field is important in calculating the photocurrent cross-section also in the case of BAs.This fact had been also observed by Bagchi and Kar [5] in the case of tungsten. Theyhave found that vector potential component in the surface region decreases greatly near the plasma frequency $\omega_{p}$ of the solids. This explain the decrease in normal photoemission from the surface states near $\omega_{p}[5]$.

\section{Conclusions}

From photoemission study of BAs, we found that the behaviour of photocurrent from the first and second layers are similar in nature, although occurrence of maxima in photocurrent is at different photon energies. Occurrence of peaks can be correlated with the peaks in density of states of electron from various energy levels with the help of density functional theory. Works in this direction is in progress which will be reported soon.

\section{Acknowledgements}

$\mathrm{RL}$ is grateful to UGC (Delhi) for the award of NFHE Fellowship, whereas RKT acknowledges a grant from SERB (DST, India) via grant No. EMR/2015/001407, Dt.11 March 2016. MPG thanks Alexander von Foundation, Germany for the award of George Foster Research Fellowship (HERMES).AHR acknowledges the CENTEM project, reg. no. CZ.1.05/2.1.00/03.0088, co-funded by the ERDF as part of the Ministry of Education, Youth and Sports OP RDI programme and, in the follow-up sustainability stage, supported through CENTEM PLUS (LO1402) by financial means from the acknowledges the Ministry of Education, Youth and Sports under the "National Sustainability Programme I. AHR Also the MetaCentrum (LM2010005) and CERIT-SC (CZ.1.05/3.2.00/08.0144) infrastructures.

\section{References}

[1] Mujica, A., Angel Rubio, A., Munoz, A. and Needs, R. J. 2003. "High Pressure Phases of Group-IV, III-V and 
II-VI Compounds.” Rev. Mod. Phys. 75: 863-912.

[2] Blaha, P., Schwarz, S., Madsen, M. K. H., Kvasnicka, D., Luitz, J. and Schwarz, K. 2015. "An Augmented Plane Wave plus Local Orbitals Program for calculating Crystal Properties: Wien $2 k$ User's Guide (Wien: TechnischeUniverstat Wien).

[3] Penn, D. R. 1972. "Photoemission Spectroscopy in the Presence of Absorbate Covered Surface." Phys. Rev. Letts. $28: 1041-4$.

[4] Pendry, J. B. 1976. "Theory of Photoemission." Surf. Sci. 57: 679-705.

[5] Bagchi, A. and Kar, N. 1978. "Refraction Effects in Angle-Resolved Photoemission from Surface States on Metals." Phys. Rev. B. 18:5240-7.

[6] Das, P. and Kar, N. 1995. "Photoemission Calculations Including Both Band Structure Effects and Photon Field Variation." Phys. Stat. Solid b 187: 551-6.

[7] Thapa, R. K. and Kar, N. 1995. "Photocurrent
Calculations in Beryllium Using a Local Dielectric Model." Phys. Rev. B. 51: 17980-3.

[8] Perdew, J. P., Burke, K. B. and Ernzerhof, M. 1996. "Generalized Gradient Approximation Made Simple." Phys. Rev. Letts. 77:3865-3868.

[9] Thapa, R. K., Das, P., and Kar, N. 1991. "Frequency Dependent Photoemission from Aluminium Using a Simple Local Dielectric Model." Physics Teacher 33: 21-6.

[10] Das, P., Thapa, R. K. and Kar, N. 1991. "Photoemission Calculation with a Simple Model for the Photon Field: Application to Aluminium." Modern Physics Letters B 5: 65-72.

[11] Thapa, R. K. and Davison, S. G. 1996. "Electromagnetic Fields Calculated in the Surface Region Using a Simple Dielectric Model and Application to Metals." Indian Journal of Pure and Applied Physics 34: 118-20. 